\title{
Virtual or reality: divergence between preprocedural computed tomography scans and lung anatomy during guided bronchoscopy
}

\author{
Michael A. Pritchett ${ }^{1}$, Krish Bhadra ${ }^{2}$, Mike Calcutt $^{3}$, Erik Folch ${ }^{4}$ \\ ${ }^{1}$ FirstHealth of the Carolinas and Pinehurst Medical Clinic, Pinehurst, NC, USA; ${ }^{2} \mathrm{CHI}$ Memorial Rees Skillern Cancer Institute, Chattanooga, TN, \\ USA; ${ }^{3}$ Clinical Education, Medtronic, Minneapolis, MN, USA; ${ }^{4}$ Massachusetts General Hospital, Harvard Medical School, Boston, MA, USA \\ Contributions: (I) Conception and design: All authors; (II) Administrative support: All authors; (III) Provision of study materials or patients: All \\ authors; (IV) Collection and assembly of data: All authors; (V) Data analysis and interpretation: All authors; (VI) Manuscript writing: All authors; (VII) \\ Final approval of manuscript: All authors. \\ Correspondence to: Michael A. Pritchett, DO, MPH. FirstHealth of the Carolinas and Pinehurst Medical Clinic, 205 Page Road, Pinehurst, NC 28374, \\ USA. Email: mpritchett@pinehurstmedical.com.
}

\begin{abstract}
Guided bronchoscopy offers a minimally invasive and safe method for accessing indeterminate pulmonary nodules. However, all current guided bronchoscopy systems rely on a preprocedural computed tomography (CT) scan to create a virtual map of the patient's airways. Changes in lung anatomy between the preprocedural CT scan and the bronchoscopy procedure can lead to a divergence between the expected and actual location of the target lesion. Termed "CT-to-body divergence", this effect reduces diagnostic yield, adds time to the procedure, and can be challenging for the operator. The objective of this paper is to describe the concept of CT-to-body divergence, its contributing factors, and methods and technologies that might minimize its deleterious effects on diagnostic yield.
\end{abstract}

Keywords: Bronchoscopy; lung neoplasms; movement; respiratory-gated imaging techniques; tomography; X-ray computed

Submitted Oct 23, 2019. Accepted for publication Jan 06, 2020.

doi: $10.21037 /$ jtd.2020.01.35

View this article at: http://dx.doi.org/10.21037/jtd.2020.01.35

\section{Introduction}

Guided bronchoscopy encompasses various minimally invasive endoscopic techniques to diagnose indeterminate peripheral lung nodules. Guidance methods include electromagnetic navigation bronchoscopy (ENB) (1-3), robotic bronchoscopy $(4,5)$, and augmented fluoroscopy among others $(6,7)$.

ENB has been the most well-studied of these technologies to date. Two main ENB systems are commercially available, the superDimension ${ }^{\mathrm{TM}}$ navigation system (Medtronic, Minneapolis, MN) and the SPiN Thoracic Navigation System $^{\text {TM }}$ (Veran Medical Technologies, St. Louis, MO). The diagnostic yield of ENB has been evaluated in over 30 clinical studies with over 3,000 combined patients, with the vast majority using the superDimension ${ }^{\mathrm{TM}}$ navigation system. Most studies report diagnostic yields in the range of $67 \%$ to $84 \%$ across both ENB platforms (8). In the prospective, multicenter, NAVIGATE study, the 1-year diagnostic yield of the superDimension ${ }^{\mathrm{TM}}$ navigation system was $73 \%$ in over 1,000 patients with a pneumothorax rate of $4.3 \%$ (2.9\% with chest tube) (2). A retrospective registry study with limited follow-up reported a much lower yield (AQuIRE) (9), although these results are inconsistent with the larger body of literature. Early data on robotic bronchoscopy is also now available. The diagnostic yield of the Ion ${ }^{\mathrm{TM}}$ endoluminal robotic system (Intuitive Surgical, Sunnyvale, CA) was recently reported at $79.3 \%$ in a 29-subject safety and feasibility study (5). A preliminary multicenter pilot study presented in abstract form on the Monarch $^{\mathrm{TM}}$ Platform (BENEFIT; Auris Surgical Robotics, Redwood City, CA) demonstrated that lesion localization was confirmed with rEBUS in 22/24 (91.7\%) patients (10) while a larger retrospective abstract including 82 patients 


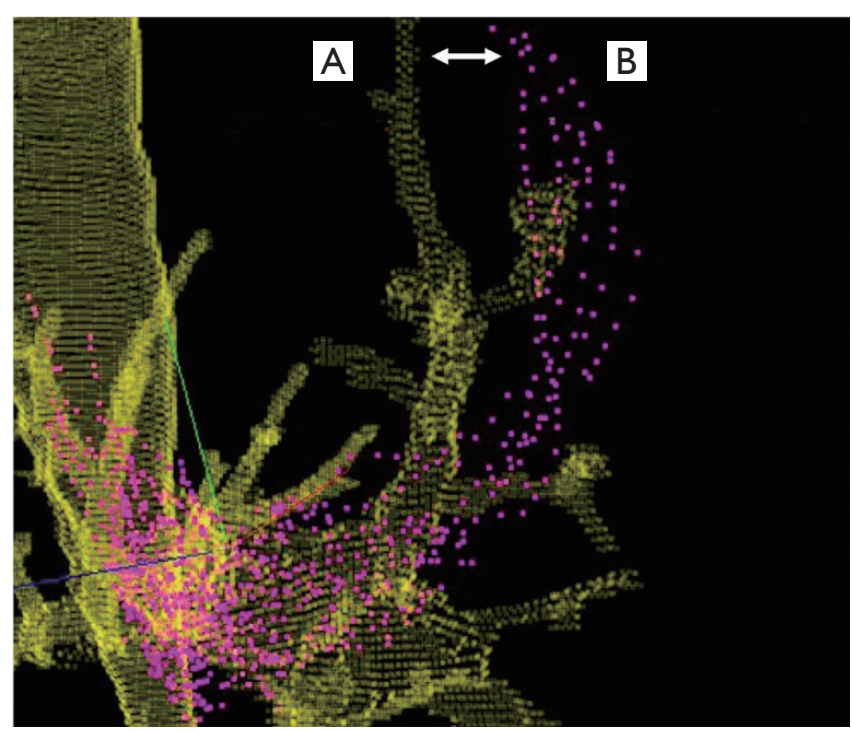

Figure 1 CT-to-body divergence. The difference between the lung map created from preprocedural CT data (A, in green) and the actual patient anatomy ( $\mathrm{B}$, in purple) is CT-to-body divergence (white arrow). All rights reserved. Used with the permission of Medtronic. CT, computed tomography.

reported navigation success in $93.9 \%$ and concentric and eccentric rEBUS confirmation in $45.7 \%$ and $38.3 \%$, respectively. The pneumothorax rate was reported at $4.8 \%$ and bleeding was reported in $3.6 \%$ (11). A peer-reviewed feasibility first-in-human study reported tissue acquisition 93\% (14/15) (4), but did not use rEBUS or electromagnetic navigation, only visual navigation. The LungPoint ${ }^{\mathrm{TM}}$ virtual bronchoscopic navigation (VBN) system (Broncus Medical, San Jose, CA) has reported diagnostic yields of $80 \%$ and $77.9 \%(12,13)$ while the Archimedes ${ }^{\mathrm{TM}} \mathrm{VBN}$ system (Broncus Medical) reported correlation with surgical histology samples in 10/12 (83\%) (6). In a 200-patient prospective multicenter study of the LungVision ${ }^{\mathrm{TM}}$ (BodyVision Medical Inc, New York, NY) augmented fluoroscopy system, also only available in abstract form, the diagnostic yield was $78 \%$ (14), with yields ranging from $75 \%$ to $87.4 \%$ in other smaller studies (15-17). These studies, while encouraging, all suffer from methodological limitations inherent in their single-arm non-comparative designs. Furthermore, the definition of "diagnostic yield" varies substantially across studies, making comparisons difficult.

In contrast to the guided bronchoscopy studies described above, diagnostic yield of transthoracic computed tomography (CT)-guided biopsy ranges from $67 \%$ to $98 \%$ with a pooled yield of $92.1 \%$ (18), though potentially lower in lesions $\leq 15 \mathrm{~mm}$ in diameter (19). While CT-guided biopsy has shown a consistently higher diagnostic yield (20), studies on TTNB arguably suffer from the same methodological limitations described in bronchoscopic studies. In addition, complication rates are higher with transthoracic biopsy than with bronchoscopic approaches (20). The respective diagnostic utility of advanced bronchoscopy and CTguided biopsy remains somewhat unclear at this time, and prospective randomized studies are needed.

A major limitation common to all guided bronchoscopic techniques is the use of a preprocedural thoracic CT scan to create a virtual map of the lesion location. CT images used in planning the virtual navigation pathway are acquired at full inspiration or inspiratory reserve volume in an awake patient, often days or weeks before the procedure. Changes in lung anatomy occur for a variety of reasons, but are primarily due to significant differences in lung volumes in sedated and sometimes mechanically ventilated patients, resulting in so-called "CT-to-body divergence". The objective of this paper is to review the concept of CT-tobody divergence, the factors contributing to its occurrence, and possible solutions to limit its impact on diagnostic yield.

\section{What is CT-to-body divergence?}

CT-to-body divergence is the difference between the static preprocedural CT reconstructions and the dynamic, breathing lung during the bronchoscopic procedure (Figure 1). Changes in lung anatomy between the preprocedural CT scan and the bronchoscopy procedure can lead to a divergence between the expected and actual location of the target lesion. During the navigation planning phase, a previously acquired CT dataset is loaded into the navigation software and the physician selects the lesion and creates a virtual path to the lesion. A registration process is then typically performed to align virtual images with the patient's actual anatomy, although this process is automated in some platforms. Despite these adjustments, the operator must still navigate to a virtual lung lesion that may be in a different location compared to the preprocedural CT. Simply stated, the problem is one of virtual versus reality. As will be described below, a variety of other procedural and anatomical factors can further aggravate this mismatch in the location of that virtual target relative to the actual lung lesion. Because there are many potential contributing factors, CT-to-body divergence is expected to be a 


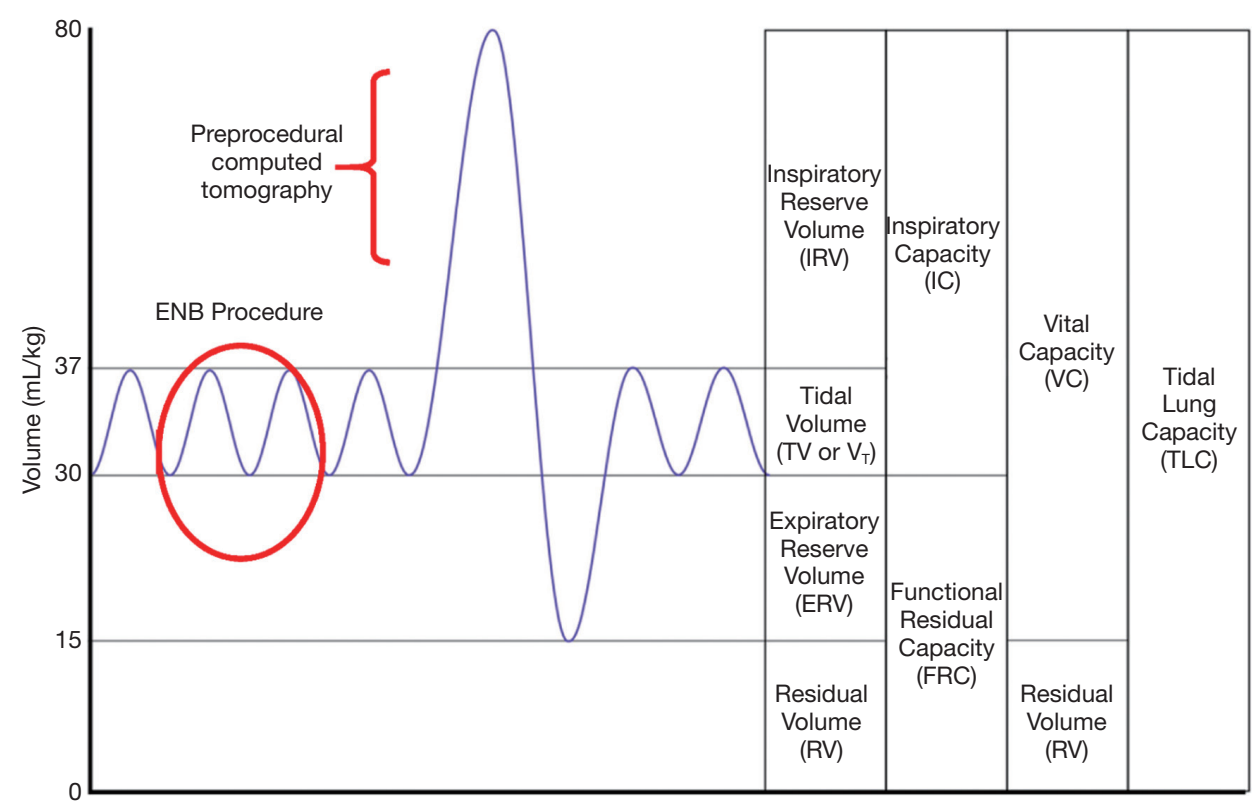

Figure 2 Lung volumes during preprocedural CT and advanced bronchoscopy procedures. Adapted from Kapwatt at English Wikipedia (https://commons.wikimedia.org/wiki/File:Lungvolumes_Updated.png) and used with permission under the terms of Creative Commons License CC BY-SA 3.0 (https://creativecommons.org/licenses/by-sa/3.0). CT, computed tomography.

pervasive problem across all platforms that rely solely on a preprocedural CT for guidance. In addition to technology improvements, an awareness of these factors and the adoption of procedural techniques to minimize CT-to-body divergence have the potential to improve diagnostic success and inform future research.

\section{Pre-procedural factors contributing to CT-to- body divergence}

\section{Lung volume differences}

The airway network is often significantly impacted by changes in lung volume between the preprocedural CT and the bronchoscopy procedure. Preprocedural CT scans are ideally conducted during a patient-initiated full inspiratory breath in order to maximize the visibility of small airways (21), at which time the chest wall and ribs are expanded and lateralized. Full expansion of the airways during the preprocedural CT scan is a vital contributor to airway mapping accuracy in several advanced bronchoscopy systems, including robotic platforms that rely on a robust three-dimensional bronchial tree. However, guided bronchoscopy is typically conducted during tidal volume breathing, when lung volume approaches functional residual capacity (Figure 2). If there is significant post-intubation atelectasis (22), the patient could be breathing at below the tidal volume somewhere near the expiratory reserve volume. In short, there is potential for substantial mismatch between the three-dimensional virtual map and the patient's actual anatomy. This has been demonstrated in a study of dynamic magnetic resonance imaging synchronized with spirometry which found a correlation between the amplitude of movement of the diaphragm during tidal breathing and lung volume. Furthermore, the investigators found that patients were capable of voluntarily moving their diaphragm, with varying amplitudes from person to person (23). This is also affected by position and spinal anatomy. In another study, the movement of a peripheral nodule between full inspiration and full expiration averaged $17.6 \mathrm{~mm}$, ranging from $<6$ to $30 \mathrm{~mm}$ in the upper lobes and $<6$ to $60 \mathrm{~mm}$ in the lower lobes (24). While one may expect that the relative positions of nodules and airways should be maintained throughout the respiratory cycle, evidence demonstrates that diagnostic yields do in fact decrease substantially in lower lobe nodules, suggesting that spatial relationship varies through the changes in lung volumes of the respiratory cycle (25).

In our experience, the airway and lesion tend to be more synchronized in lateral segments. In straight basilar airway segments (particularly in the posterior dependent lung) the 

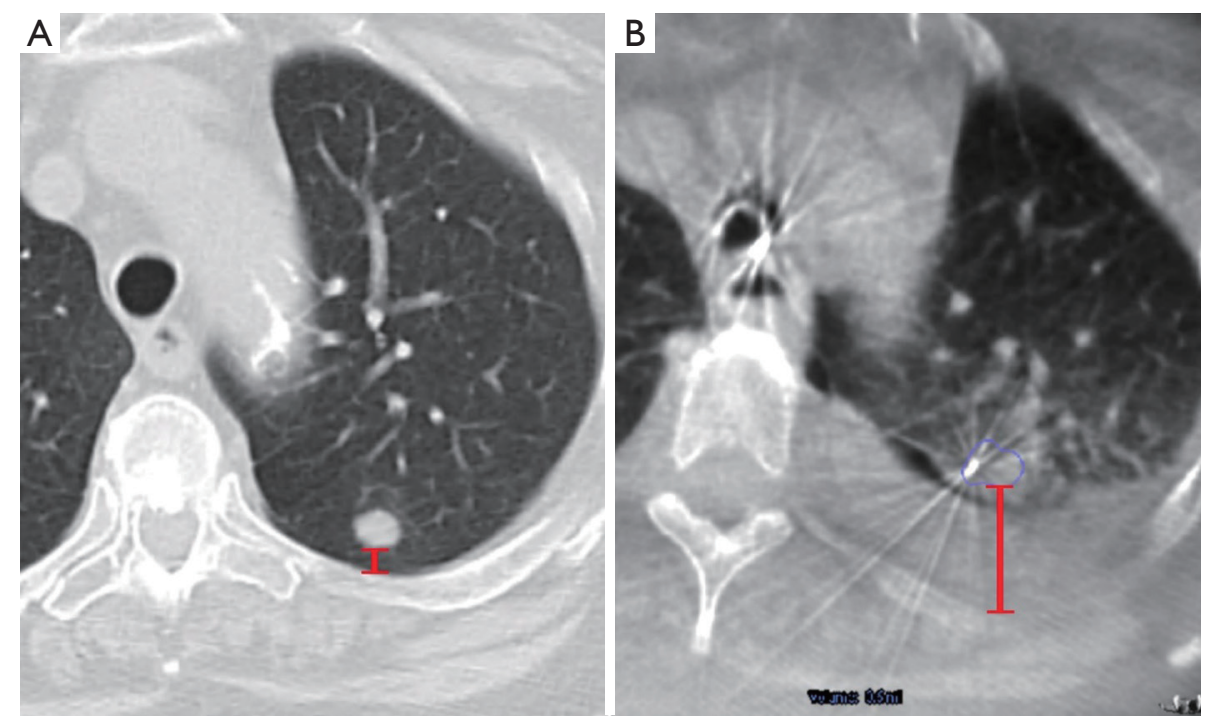

Figure 3 Pleural effusion causing divergence. Preprocedural CT scan taken 7 days prior to the procedure (A) and an intraoperative CBCT scan (B). The patient developed congestive heart failure and small pleural effusion, causing significant CT-to-body divergence (red bar). Image courtesy of Dr. Michael Pritchett. CT, computed tomography. CBCT, cone beam CT.

lesion location may vary greatly during respiration and the catheter or scope can slide in and out of the airway, causing more divergence. This is further supported by the dynamic MRI studies showing the increased displacement of the posterior basal segments that are in proximity to the caudal part of the diaphragm (23).

Each manufacturer of advanced bronchoscopy equipment has different recommendations for preprocedural CT protocols and timing (26). The SPiN Thoracic Navigation System $^{\mathrm{TM}}$ recommends a full inspiratory scan obtained with the arms above the head followed by a full expiratory scan with the arms at the patient's side (26). This system uses an algorithm to pair inspiratory and expiratory CT scans with the respiratory cycle in order to compensate for respiratory variation, in an attempt to mitigate CT-to-body divergence. One study found that the inspiratory/expiratory CT scan protocol with periprocedural respiratory motion sensing and gating provides better nodule location accuracy than inspiratory-only CT scans (21). However, while intuitively appealing, limited data are currently available on this system, with reported diagnostic yields of 33\% (27), 90\% (28), and $83 \%$ (3) in three small, single-center studies. One potential reason for the lack of a notable benefit is that in the far periphery, the airway (and thus the catheter) often moves in synchrony with the nodule. Another potential downside is the increased radiation exposure from the sameday inspiratory and expiratory CT scans. The inspiratory/ expiratory protocol resulted in a radiation dose of $9.5 \mathrm{mSv}$ in one study (29). A multicenter prospective trial is ongoing to more fully evaluate this technology and the impact on diagnostic yield (NCT03338049) (30).

\section{Preprocedural CT scan timing and parameters}

The time delay between the preprocedural CT scan and bronchoscopy can also affect the relative accuracy between the virtual map and the actual lung anatomy. Preprocedural CT scans are often conducted days, weeks, or even months prior to the procedure, although the currently available systems recommend the preprocedural CT be conducted as close to the procedure data as possible; the SPiN Thoracic Navigation System ${ }^{\mathrm{TM}}$ recommends a same-day scan.

Interestingly, two studies showed that in approximately $7-10 \%$ of cases, the lung lesion noted on the initial CT scan can actually decrease in size or disappear prior to the bronchoscopy $(29,31)$. In a study of the Veran system, $6.9 \%$ of nodules decreased in size or resolved compared to the initial CT, which averaged 53 days prior to the ENB procedure (29). Pleural effusions (Figure 3) and mucus plugs can also develop or resolve between the preprocedural $\mathrm{CT}$ and the procedure, causing changes or distortions. Conducting preprocedural CT scans close to or on the same day as the guided bronchoscopy can potentially reduce CTto-body divergence related to time delays, although with a 
higher cost to the healthcare system and increased radiation exposure.

It is also important that the reconstruction parameters of the CT scan are optimized to provide an adequate source for the virtual map. Each manufacturer has specific recommended parameters for slice thickness, slice interval, percent overlap, and kernel (26). If the preprocedural CT scan is not optimized, a new CT scan with the correct parameters should be repeated prior to the guided- bronchoscopy, although financial cost and patient compliance may be obstacles.

While following these recommendations may mitigate CT-to-body divergence, lung volume, reconstruction parameters, and the timing of the preprocedural CT are not the only contributing factors. Even with same-day optimized CT scans, anatomical changes can be introduced before and during the bronchoscopic procedure. These procedural and anatomical factors can potentially introduce error with all guided-bronchoscopy systems that rely solely on preprocedural CT scans.

\section{Anatomical changes}

Other anatomical changes can occur during guided bronchoscopy due to patient positioning. The preprocedural CT scan is typically taken with the patient's arms overhead. Thoracic anatomy will therefore be different during the procedure with the patient in a supine position with the arms at the side. Bed arrangement (curved versus flat) and the use of a head pillow during the preprocedural CT can also change the thoracic anatomy and lead to a mismatch between the preprocedural CT scan and the actual patient anatomy during the procedure. Arching of the upper back during the intubation process can create small fluctuations in airway anatomy, leading to inaccurate localization of the target nodule during the registration process. It is also important to ensure that the configuration of the bed, location board, and field generator are set up as instructed by the manufacturer to provide the most accurate virtual map.

\section{Ferromagnetic objects}

In some systems reliant on an electromagnetic field (i.e., superDimension $^{\mathrm{TM}}$ navigation system, SPiN ${ }^{\mathrm{TM}}$ Thoracic Navigation System, Monarch ${ }^{\mathrm{TM}}$ platform), ferromagnetic materials can cause distortions when placed within the range of an electromagnetic field. Therefore, it is important to follow the manufacturer's instructions regarding factors that affect the magnetic field, such as anesthesia equipment, intravenous poles, fluoroscopy units, beds, and other ferromagnetic objects.

\section{Procedural factors contributing to CT-to-body divergence}

\section{Registration technique}

The registration process aligns the preprocedural CT scan to the patient's body to create a three-dimensional map of the lungs. Most guided-bronchoscopy systems use a centrally based registration process, at least during the initial registration phase, and therefore may be less accurate in the periphery. Each manufacturer recommends techniques to optimize this registration process to ensure the most accurate representation of both central and peripheral airways.

\section{Atelectasis}

Even after successful registration and creation of the virtual lung map, anatomical changes can occur and evolve during the guided-bronchoscopy procedure. Atelectasis can cause an inaccurate virtual lung map, and also decrease visibility of the lesion on imaging modalities (Figure 4). Atelectasis is an often underappreciated issue, despite literature reports that atelectasis occurs in $87-100 \%$ of healthy adults within minutes of general anesthesia induction (32-34). Atelectasis is exacerbated in obese individuals and those with scoliosis; therefore, use of a specific ventilation strategy to avoid this issue $(35,36)$ may be the difference between success or failure in diagnosing these lesions. This may be especially important for lesions in an inferior or posterior position, where atelectasis is most likely to occur.

Atelectasis can be caused by prolonged time to intubation, suboptimal ventilation protocols, high fractions of inspired oxygen leading to absorption atelectasis and, importantly, distal wedging of the bronchoscope. Increasing use of conebeam CT (CBCT) has heightened physicians' awareness of anesthesia-induced atelectasis and the impact on lung anatomy. In one study, atelectasis was identified by CBCT in $40 \%$ of cases overall and was found to completely obscure the target in $20 \%$, resulting in a lower diagnostic yield (only $50 \%$, prior to correction based on CBCT data) (22) than observed in other studies of CBCT-guided bronchoscopy (37). The type of anesthesia (general anesthesia versus moderate sedation), and patient body habitus (i.e., obesity, spinal anatomy) are 

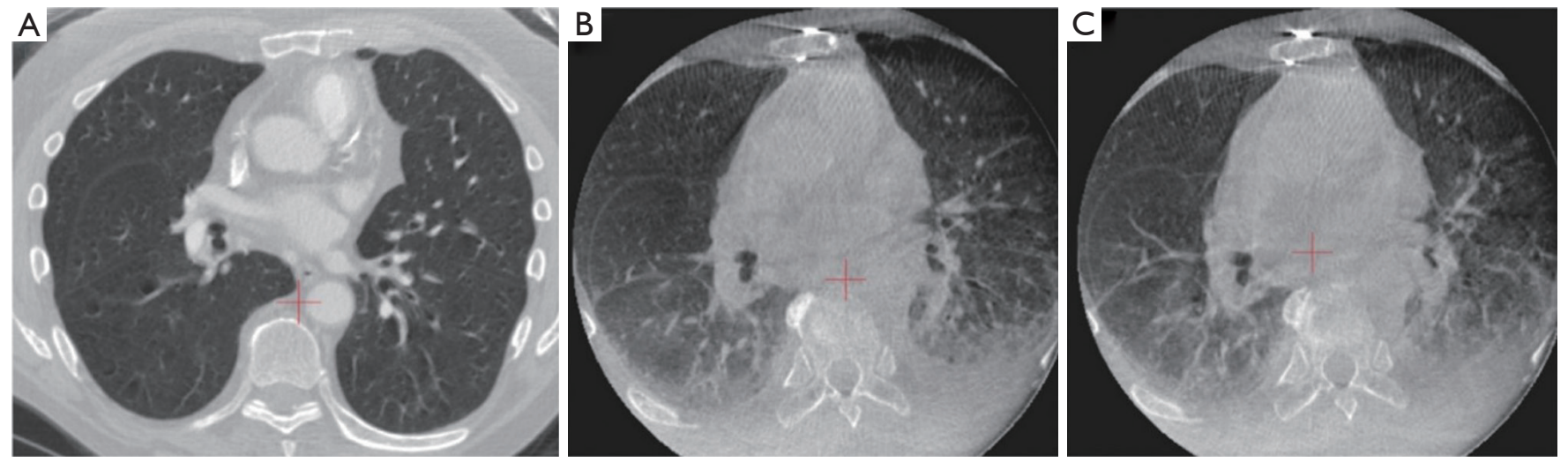

Figure 4 Atelectasis. (A) Preprocedural CT scan; (B) CBCT immediately after induction of anesthesia (before beginning the bronchoscopy procedure); (C) CBCT scan conducted at the end of the procedure. This example highlights that atelectasis can begin early in the procedure and does not resolve. Image courtesy of Dr. Michael Pritchett. CT, computed tomography. CBCT, cone beam CT.

also likely significant contributors. If the procedure is done under moderate sedation, it becomes challenging to predict the respiratory mechanics that may influence CT-to-body divergence.

Atelectasis can also be caused by the bronchoscopic tools and manipulation of the airways during the procedure. One common issue is that the bronchoscope can be wedged in the airway, creating an obstruction that causes collapse of the tissue (obstructive atelectasis) and possibly distortion of the airways beyond the bronchoscope. This is further exacerbated by applying suction. The extended working channel and endoscopic tools used during lung nodule sampling can also displace the anatomy and cause distortion of the airways, depending on the flexibility of the catheters and instruments used. Staging of the mediastinum before sampling the lung nodule, a common practice among advanced bronchoscopists and interventional pulmonologists based on current guidelines, also extends the time that the patient is supine and under anesthesia. This may lead to de-recruitment of the peripheral alveoli and more atelectasis, particularly in dependent areas of the lung or near the diaphragm. Atelectasis and anatomical changes in the airways can progress during the bronchoscopic procedure, creating a dynamically changing airway structure that no longer matches the original virtual map. While more common in the lower lobes, these effects can occur in the upper lobes as well (24). More flexible catheters and instruments may minimize deflection of the airways, though atelectasis can still occur and is an important challenge that users must understand. Many experts now recommend that navigation bronchoscopy be conducted prior to mediastinal staging to minimize the cumulative effects of CT-tobody divergence during the procedure, provided there is no obvious evidence of mediastinal metastasis. Optimized ventilation protocols, minimizing unnecessary suctioning, and avoiding over-wedging of the scope may significantly minimize atelectasis (see Table 1). Figure 5 shows two cases before (A) and after (B) the induction of a specific ventilation protocol designed to prevent atelectasis.

\section{Chest muscle changes}

Since lung motion can reduce image quality in imageguided bronchoscopy, some technologies, such as CBCT and fluoroscopic navigation, recommend the use of paralytics. Neuromuscular blocking agents act on the effector muscles, most profoundly on those of the upper airway (42). The nondependent (top) part of the lung has been shown to be most displaced after neuromuscular blockade and positive pressure ventilation (43). Paralytics have little effect on the diaphragm in supine patients and a greater effect on the abdominal muscles $(42,44,45)$, hence the aforementioned ventilation strategies are important to minimize lung motion in addition to the use of paralytics. Relaxation of the expiratory abdominal muscles and intercostal muscles (42) changes the conformation of the chest wall and may cause CT-to-body divergence. Therefore, these findings need to be taken into account when using paralytics and inhaled anesthetics. Further research into anesthesia and ventilation protocols optimized to the technology will be important to control the chest muscle factors that contribute to CT-tobody divergence. 
Table 1 Ventilation best practices for guided bronchoscopy

- Rapid intubation (not rapid sequence intubation) to minimize atelectasis. If intubation was prolonged due to difficult airway, recruitment maneuvers are recommended. Use of continuous positive airway pressure therapy (CPAP)/positive end expiratory pressure (PEEP) during the pre-oxygenation/induction process has been shown to significantly minimize atelectasis

- An endotracheal tube is preferred over the laryngeal mask airway (LMA) in patients undergoing guided bronchoscopy procedures for peripheral nodules. Our recommended ventilation strategy (outlined below) employs higher airway pressures (larger tidal volumes and increased PEEP). Therefore, an endotracheal tube can better ensure maintenance of these pressures. Additionally, there is risk of increased gastric insufflation when using the LMA with higher pressures. This in turn may increase the risk for aspiration, although the risk remains low based on previous studies (38). However, this recommended ventilation strategy can also be done with an LMA; consultation with the local anesthesia department is advised (39)

- Jet ventilation has also been used with some success to minimize lung motion and mitigate the factors contributing to CT-to-body divergence during guided bronchoscopy procedures $(40,41)$. However, use of this method may be limited by availability and expertise

- Avoid hyperoxia. Inspired oxygen content should be kept as low as tolerated to minimize absorption atelectasis during the procedure [target range for initial fraction of inspired oxygen $\left(\mathrm{FiO}_{2}\right): 30-40 \%$ ]. The use of $80 \% \mathrm{FiO}_{2}$ has also been used during the induction phase to minimize atelectasis (35)

- Judicious use of PEEP from the pre-induction phase and throughout the procedure $(35,36)$. Usual levels are $8-15 \mathrm{cmH}_{2} \mathrm{O}(34,36)$, but may vary based on patient characteristics (morbid obesity, lower lobe/posterior lesions may require higher levels)

- Use higher tidal volumes, typically $10-12 \mathrm{cc} / \mathrm{kg}$ of ideal body weight. Jet ventilation has also been used with success, if it is available

- Use of paralytics when using general anesthesia is recommended, particularly if using tomosynthesis or CBCT which require breath hold maneuvers

- Use of the adjustable pressure-limiting (APL) valve during breath hold maneuvers is critical. This should be set between $10-40 \mathrm{cmH}_{2} \mathrm{O}$ (depending on patient factors and nodule position) to avoid elevation of the diaphragm and atelectasis during breath holds. Waiting 5-8 seconds after breath hold before starting CBCT or tomosynthesis spin can help to minimize residual motion artifact
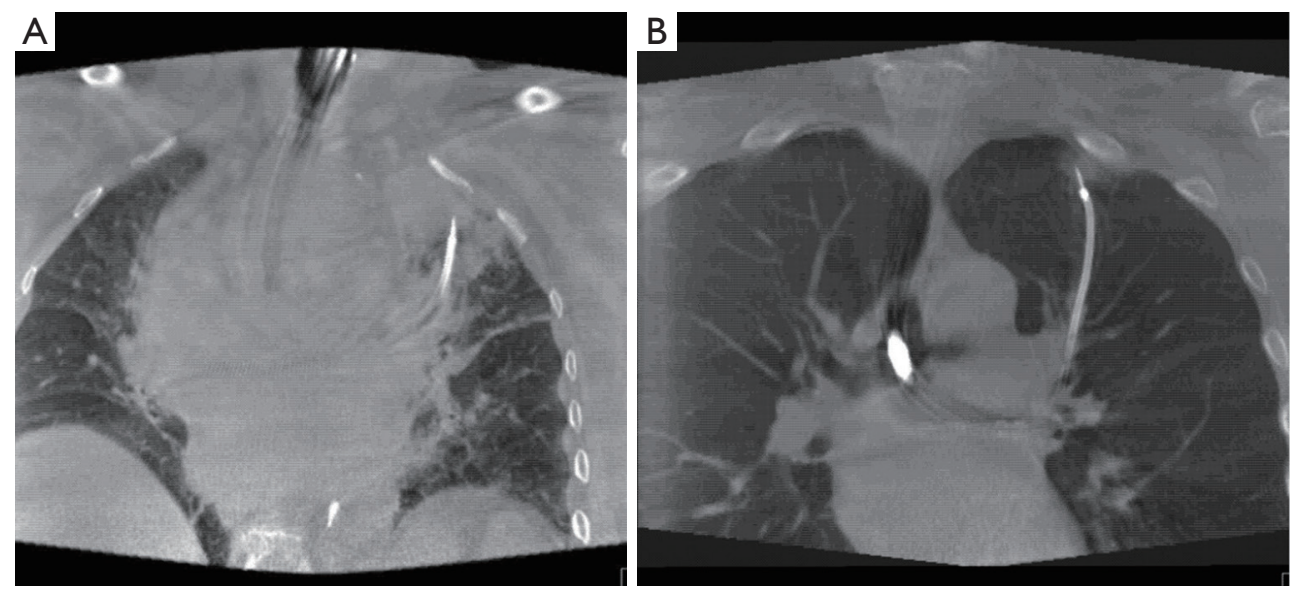

Figure 5 Two different cases before (A) and after (B) the introduction of a specific ventilation protocol designed to prevent atelectasis. On the left, the preprocedural CT scan was taken one week prior to the procedure, yet the lesion is entirely obscured by atelectasis with a significant ghosting artifact. Image courtesy of Dr. Krish Bhadra. CT, computed tomography.

\section{Minimizing CT-to-body divergence}

Changes in lung anatomy can occur for a variety of reasons between the preprocedural CT and the guided bronchoscopy procedure. It is critical to recognize that the virtual target is not necessarily in the same location as the real lesion, and to understand the factors that may contribute to such discrepancies. As discussed above, several best practice techniques can minimize CT-to-body divergence. However, despite the most careful technique, many factors are outside of the user's control. For that reason, systems are being developed to help compensate for CT-to-body divergence (Table 2). 
Table 2 Guided bronchoscopy platforms

\begin{tabular}{|c|c|c|c|}
\hline Product & Manufacturer & Technology & $\begin{array}{l}\text { Peer-reviewed journal } \\
\text { publications }\end{array}$ \\
\hline $\begin{array}{l}\text { superDimension }^{\mathrm{TM}} \\
\text { navigation system } \\
\text { (version } 7.1 \text { and } \\
\text { below) }\end{array}$ & Medtronic & $\begin{array}{l}\text { Electromagnetic tracking with a steerable } \\
\text { locatable guide and working channel }\end{array}$ & $\begin{array}{l}\text { Over } 100 \text { original research } \\
\text { articles to date representing data } \\
\text { from over } 75 \text { clinical studies }(2,8)\end{array}$ \\
\hline $\begin{array}{l}\text { SPiN Thoracic } \\
\text { Navigation System }^{\mathrm{TM}}\end{array}$ & Veran Medical & $\begin{array}{l}\text { Based on an external electromagnetic generator, } \\
\text { uses tip-tracked instruments for continuous } \\
\text { guidance in a trackable airway map, an } \\
\text { inspiration/ expiration computed tomography } \\
\text { (CT) scan protocol, and an algorithm to pair } \\
\text { inspiratory and expiratory CT scans with the } \\
\text { respiratory cycle in order to compensate for } \\
\text { respiratory variation (respiratory gating)* }\end{array}$ & $\begin{array}{l}4 \text { clinical studies }(3,27,28,47) \\
\text { and } 1 \text { case report }(48) \text { on } \\
\text { guided bronchoscopy with } \\
\text { the SPiNDrive system }{ }^{\mathrm{TM}} \text { for } \\
\text { navigated bronchoscopy*. } \\
\text { "All-in-One" study currently } \\
\text { recruiting (30) }\end{array}$ \\
\hline $\begin{array}{l}\text { LungPoint }{ }^{\mathrm{TM}} \text { virtual } \\
\text { bronchoscopic } \\
\text { navigation (VBN) } \\
\text { system }\end{array}$ & Broncus Medical & $\begin{array}{l}\text { Image-based synchronization technique (partly } \\
\text { manual). No registration of integrated tracking } \\
\text { method }\end{array}$ & $\begin{array}{l}\text { Eberhardt et al. } 2010 \text { (12), Tamiya } \\
2013 \text { (13), and Sterman et al. } \\
2015 \text { (49) }\end{array}$ \\
\hline Bf-Navi & Olympus, Tokyo, Japan & $\begin{array}{l}\text { Virtual bronchoscopy. No integrated tracking } \\
\text { method }\end{array}$ & $\begin{array}{l}\text { Oki et al. } 2019 \text { (50), Ali et al. } \\
2019 \text { (51) }\end{array}$ \\
\hline $\begin{array}{l}\text { Lung Suite, Cone- } \\
\text { Beam Computed } \\
\text { Tomography }\end{array}$ & $\begin{array}{l}\text { Philips, Best, The } \\
\text { Netherlands }\end{array}$ & $\begin{array}{l}\text { Overlays three-dimensional CBCT data on } \\
\text { live fluoroscopy (augmented fluoroscopy) with } \\
\text { automatic positional adaptation }\end{array}$ & $\begin{array}{l}\text { Hohenforst-Schmidt et al. } \\
2014 \text { (53), Pritchett et al. } \\
2018 \text { (37), Ali et al. } 2019 \text { (51), } \\
\text { Sobieszczyk et al. } 2018 \text { (54), } \\
\text { Bowling et al. } 2017 \text { (55) }\end{array}$ \\
\hline $\begin{array}{l}\text { lon }{ }^{\mathrm{TM}} \text { endoluminal } \\
\text { robotic system }\end{array}$ & Intuitive Surgical & $\begin{array}{l}\text { Uses direct continuous visualization and fiber- } \\
\text { optic, real-time shape-sensing technology }\end{array}$ & Fielding et al., 2019 (5) \\
\hline Monarch $^{\text {TM }}$ Platform & Auris Surgical Robotics & $\begin{array}{l}\text { Electromagnetic-based. Uses "fused navigation" } \\
\text { of multiple data modalities (electromagnetic } \\
\text { navigation, direct visualization, real-time optical } \\
\text { pattern recognition, machine learning) to integrate } \\
\text { the preprocedural CT into an intraprocedural } \\
\text { interface }\end{array}$ & $\begin{array}{l}\text { REACH study (56), Rojas-Solano } \\
\text { et al. } 2018(4), \text { two abstract } \\
\text { reports }(10,11)\end{array}$ \\
\hline
\end{tabular}

*The SPiN Thoracic Navigation System ${ }^{\mathrm{TM}}$ also includes SPiN Perc ${ }^{\mathrm{TM}}$, a system of navigated transthoracic needle aspiration (27,57-59) which is outside the scope of this review article yet still subject to CT-to-body divergence. 


\section{Tip-tracked instruments and catbeters}

Catheters and biopsy tools with embedded tracking devices have the potential to improve diagnostic yield. The chest CT protocol used with the SPiN Thoracic Navigation System $^{\mathrm{TM}}$ employs navigational tracking pads that remain in place during the bronchoscopy procedure to create a trackable virtual airway map with automatic registration and dynamic referencing (57). Electromagnetic tip-tracked instruments are then used throughout the procedure to continuously track the path to the target. A study of 93 subjects undergoing lung lesion biopsy with the tip-tracked ENB system combined with ENB-guided TTNA reported a diagnostic yield of $78 \%$ (74\% with ENB alone) (47). In another multicenter study of 129 patients the diagnostic yield was 74\% with ENB-guided TTNA and $81 \%$ when combined with ENB-guided bronchoscopic sampling, with a pneumothorax rate of $18 \%$ (12\% requiring a chest tube) (57). While always-on tip tracking has theoretical advantages, more evidence is needed on this technology.

\section{Direct visualization}

Direct visualization using thin/ultrathin bronchoscopes is used to assist with navigation in combination with rEBUS, fluoroscopy, CBCT, or virtual navigation $(51,60)$. Direct continuous visualization during navigation is also employed by both the Ion ${ }^{\mathrm{TM}}$ endoluminal robotic system and the Monarch ${ }^{\mathrm{TM}}$ platform. The Ion ${ }^{\mathrm{TM}}$ system includes a camera on a removable probe while the Monarch ${ }^{\mathrm{TM}}$ platform employs a built-in camera. Direct visualization during navigation can theoretically overcome some aspects of CT-to-body divergence by directly visualizing tumors or mucosal changes associated with early invasion of the distal airways. This method also helps to visualize additional airways that may not be visible on preoperative CT (and therefore not seen during planning phase of the procedure) and thus assists with navigating through them to get to the target.

\section{Optical pattern recognition}

In addition to direct visualization, the Monarch ${ }^{\mathrm{TM}}$ platform (Figure 6) uses "fused navigation" of multiple data modalities (electromagnetic navigation, optical pattern recognition, and robotic data) to integrate the preprocedural CT scan into an intraprocedural interface. The software algorithm continually re-registers the position of the scope within the lung as navigation proceeds. Thus, while this system still uses a preprocedural CT scan as the basis for its virtual map, real-time optical pattern recognition and machine learning are used to update the position relative to the actual lung anatomy. Evidence on the effectiveness of this method is needed; peer-reviewed data to date are limited to one small feasibility study of bronchus-sign lesions that did not evaluate diagnostic yield (4) and did not use navigation technology or rEBUS, only direct visualization.

\section{Shape-sensing}

Fiber-optic shape-sensing technology in the Ion ${ }^{\mathrm{TM}}$ endoluminal robotic system provides a real-time shape of the entire catheter, combined with direct visualization of the patient's airways during navigation (Figure 7). This system tracks the shape and motion of the lung airways with high precision and provides a complete view of the catheter in the airway, with high-speed feedback of the catheter location relative to airway anatomy during the bronchoscopy procedure. Navigation and robotic control are also active during the full procedure, including biopsy, under fluoroscopy. Encouraging results were noted in a single center first-in-human study (5) but will need to be replicated in a wider patient population. The prospective, multicenter PRECIsE study is currently enrolling, with plans to evaluate the system in up to 360 patients across 6 United States sites, including both academic and community settings (NCT03893539) (61).

\section{Real-time advanced imaging}

\section{Radial EBUS}

It has long been thought that the use of intraprocedural radial endobronchial ultrasound (rEBUS) improves location accuracy and increases diagnostic yields (25). However, a more recent multicenter, prospective, randomized study (average lesion size $=3.1 \mathrm{~cm}$ ) did not show a significant improvement in diagnostic yield with the use of rEBUS (49\%) compared to traditional bronchoscopy with fluoroscopy (37\%) (62), possibly due to the selective use of rEBUS in more challenging cases $(2,9,63)$. If the rEBUS image is not concentric, it is difficult to determine the direction of the lung lesion in space, and the diagnostic yield is reduced in such lesions (62). In one study, diagnostic yield of rEBUS was $84 \%$ when a concentric view was obtained versus $48 \%$ for eccentric views (64). Radial EBUS also does not provide real-time confirmation that the biopsy 


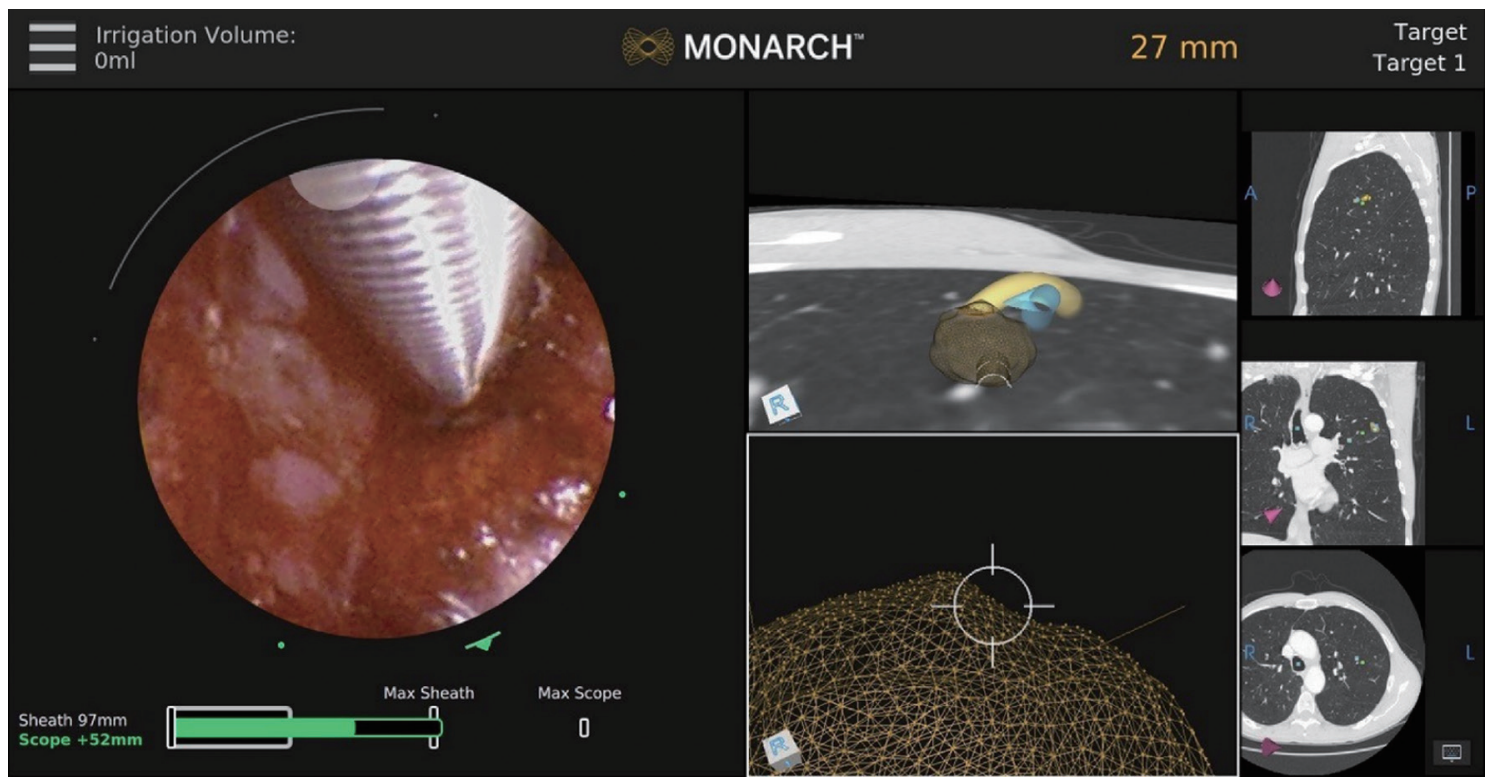

Figure 6 The Monarch ${ }^{\mathrm{TM}}$ Platform. Fused navigation of multiple data modalities is used to integrate the preprocedural CT scan into an intraprocedural interface. The software algorithm continually re-registers the position of the scope within the lung as navigation proceeds. Image courtesy of Dr. Stephen Kovacs. All rights reserved. CT, computed tomography.

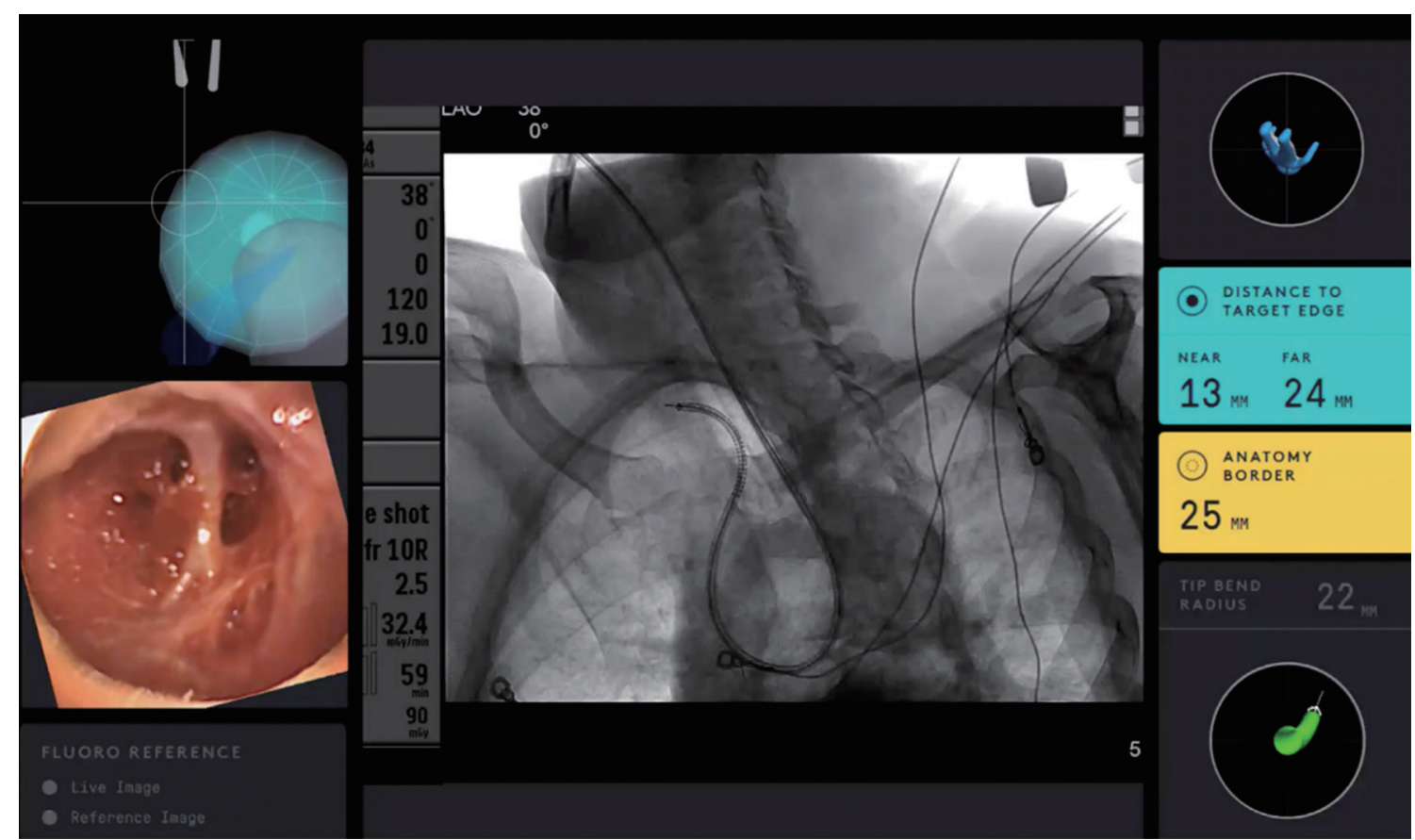

Figure 7 Ion $^{\mathrm{TM}}$ endoluminal robotic system. Fiber-optic shape-sensing technology combined with direct visualization and high-speed feedback of catheter location provide a complete view of the catheter in the airway during the bronchoscopy procedure. Image courtesy of Dr. Michael Pritchett. 


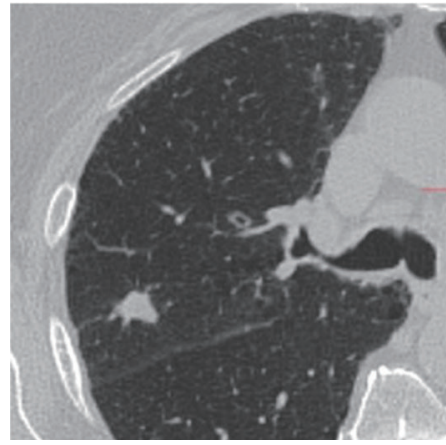

Lesion identification on $\mathrm{CT}$

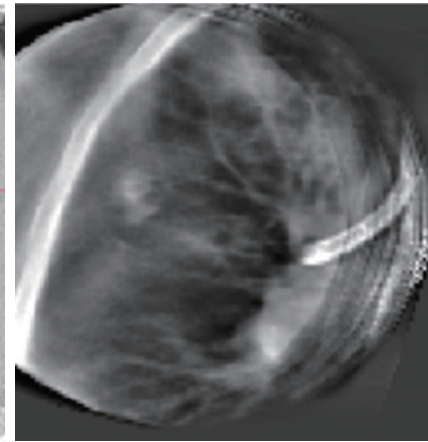

Real-time Al tomographic lesion reconstruction with $\mathrm{CABT}$ during dynamic registration

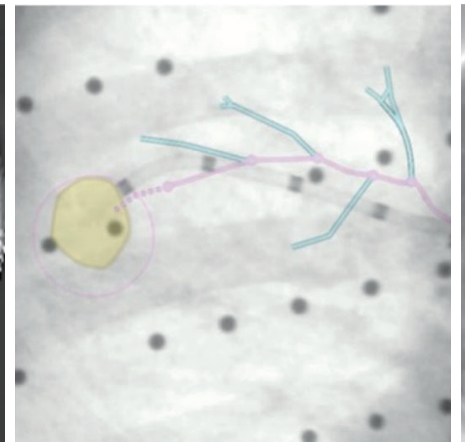

Dynamic registration and navigation along planned pathway towards the lesion

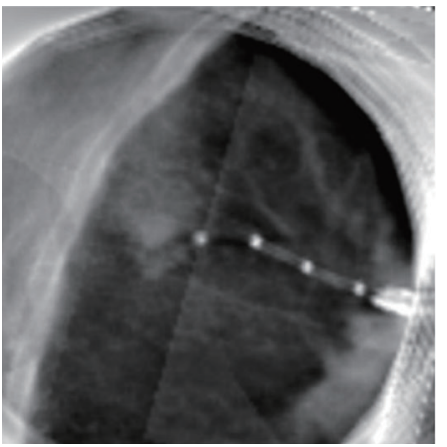

Real-time "tool in lesion" confirmation with CABT

Figure 8 Case example of LungVision ${ }^{\mathrm{TM}}$ use in a 73 -year-old female with a $15 \mathrm{~mm}$ right upper lobe lesion. LungVision ${ }^{\mathrm{TM}}$ applies dynamic registration technology, while fusing preprocedural CT scans with interoperative fluoroscopy and real-time tomographic reconstruction of the lesion from standard fluoroscopy (C-arm-based tomography, CABT) to identify the accurate location of the lesion during diagnostic procedure, while continuously compensating for the CT-to-body divergence. At the end of navigation, a CABT spin is performed to confirm tool-in-lesion and to allow guided biopsy sample collection. Image courtesy of Dr. Joseph Cicenia, Cleveland Clinic. CT, computed tomography.

tool is in the target (like convex/linear probe EBUS), as the rEBUS probe must be removed to allow for endoscopic biopsy tools to be used.

\section{Cone-beam computed tomography}

The CBCT system is compact enough to be mounted on a moving c-arm, providing complete volumetric data acquisition in a single rotation around the stationary patient. CBCT can be used alone (53) or in combination with other guidance or confirmation modalities such as radial EBUS, ENB, augmented fluoroscopy, and VBN $(22,37,51)$. A recent study evaluated forceps biopsy using ultrathin bronchoscopy, VBN, and CBCT (without augmented fluoroscopy) in bronchus sign lesions. CBCT visualized all lesions, with a diagnostic yield of $90.0 \%$ (51). In another study, CBCT (without augmented fluoroscopy) was used when a diagnosis could not be obtained using REBUS and ultrathin bronchoscopy. An increase in diagnostic yield was observed, from $50 \%$ pre-CBCT to $70 \%$ following post-CBCT maneuvers (22), although atelectasis limited the diagnostic yield. Lung Suite (Philips, Best, The Netherlands) overlays three-dimensional CBCT data on live fluoroscopy (augmented fluoroscopy). This data maintains geometric correspondence throughout the case while moving the c-arm, the table, and during magnification. With the necessary software, all CBCT systems (with the exception of mobile systems) have the ability to incorporate augmented fluoroscopy. CBCT combined with augmented fluoroscopy and the
superDimension $^{\mathrm{TM}}$ navigation system (version 7.1), resulted in a diagnostic yield of $83.7 \%$ and a diagnostic accuracy rate of $93.5 \%$ in 75 consecutive patients with a median lesion size of only $16 \mathrm{~mm}$ (37). CBCT imaging is necessary for bronchoscopic ablation procedures as it can accurately show the location of the probe, target lesion, and critical structures in real time prior to ablation. Additionally, this imaging modality is critical to assess the zone of ablation after the procedure (65). Another benefit of CBCT is that, if a diagnosis is not obtained by guided bronchoscopy, a percutaneous biopsy can be conducted based on the real-time position of the lesion (rather than the "virtual" position of the lesion).

\section{Augmented and fused fluoroscopy}

Augmented fluoroscopy may also be utilized as a standalone platform, as in the LungVision ${ }^{\mathrm{TM}}$ system (Figure 8), which uses artificial intelligence to make targeted soft lesions visible in real time with standard $\mathrm{C}$-arm during navigational bronchoscopy. This system applies dynamic registration technology to accurately identify the lesion location during the diagnostic procedure by fusing preprocedural CT scans with intraprocedural fluoroscopy and real-time tomographic reconstruction of the lesion from the standard fluoroscope (C-arm based tomography, CABT). This system is capable of dynamically tracking lesion movement during the procedure to address respiratory motion and airways distortion due to insertion of biopsy tools. Published data to date are limited to conference abstracts that show 

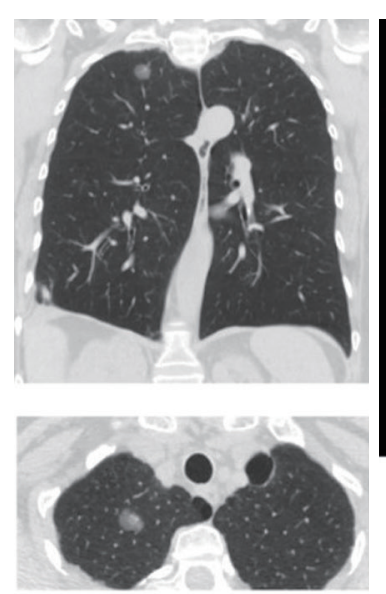

Preprocedural CT Scans

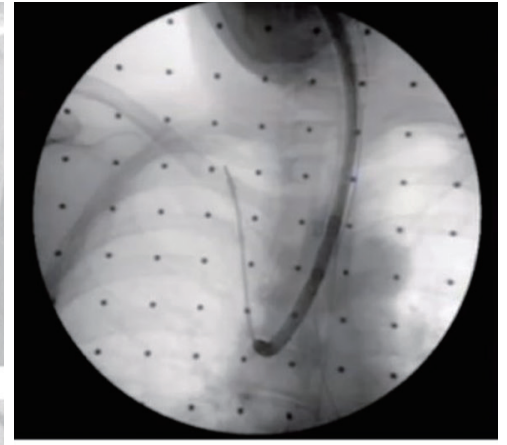

AP fluoroscopy demonstrating no visualization of the tumor during the fluoroscopic navigation workflow

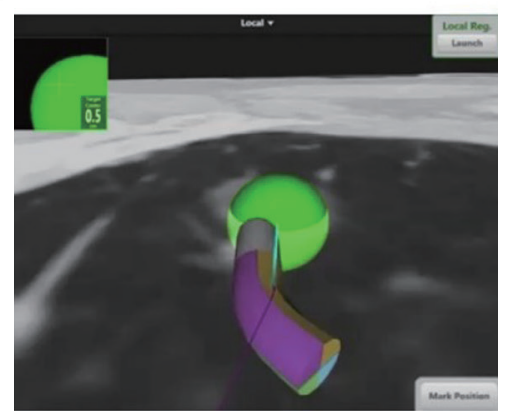

Initial navigation prior to local registration

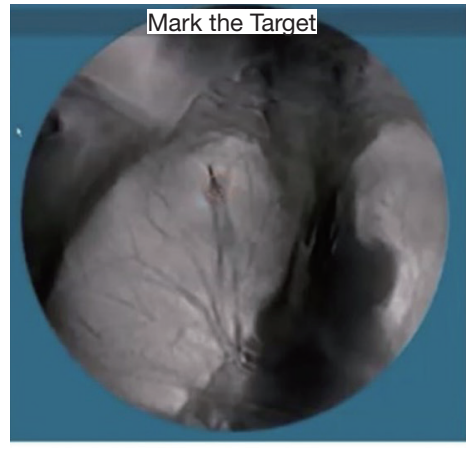

Initial volume with the navigation catheter past the lesion

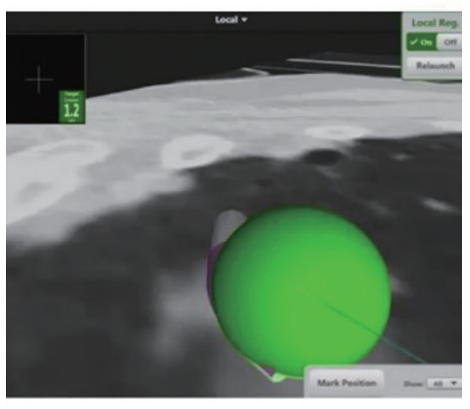

Corrected position after local registration performed

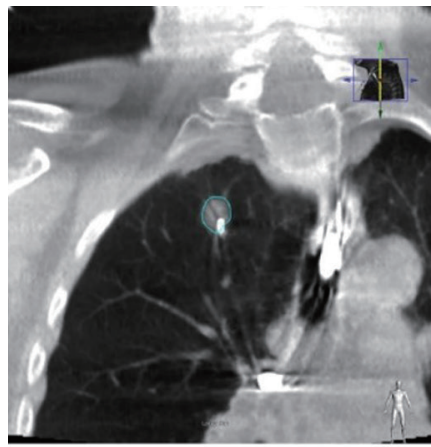

CBCT confirmation after fluoroscopic navigation correction

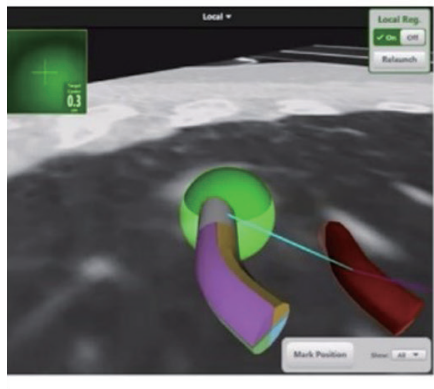

After local registration correction and realignment to the nodule

Figure 9 SuperDimension ${ }^{\mathrm{TM}}$ navigation system with fluoroscopic navigation technology. Bottom row shows system screens corresponding to each image in the top row. Fluoroscopic navigation uses advanced software algorithms and digital tomosynthesis reconstruction of multiple fluoroscopic images to enhance visualization and provide accurate three-dimensional modeling. A local registration feature employs fluoroscopy and a proprietary algorithm to update the virtual target location intraprocedurally so that the user can reposition the catheter as needed based on the actual nodule location, therefore minimizing any CT-to-divergence. CT, computed tomography.

localization rates $>90 \%$ and diagnostic yield ranging from $75-87.4 \%$ (although this data was prior to the use of CABT) (14-17). In one study, diagnostic yield of this system was $87 \%$ despite an initial CT-to-body divergence of $14.1 \mathrm{~mm}$ based on the preprocedural CT (16). Multicenter studies of the LungVision system are ongoing. The Archimedes ${ }^{\mathrm{TM}}$ system uses a unique registration board under the patient with settings specific to that patient. A specialized headrest and knee wedge also ensure consistent positioning. The same board and settings are used for both the preprocedural CT and the bronchoscopy procedure. Registration is conducted using infrared cameras and radiopaque markers to create augmented fluoroscopic views ("fused fluoroscopy") during bronchoscopic transparenchymal nodule access. This method helps to diminish positional changes between the preprocedural CT and the bronchoscopy procedure. One published study reported a diagnostic yield of $83 \%$ (6), but more research is required. Another study, EAST2, has recently been completed (66).

\section{Tomosynthesis-based fluoroscopic navigation}

The superDimension ${ }^{\mathrm{TM}}$ navigation system version 7.2 (Medtronic, Minneapolis, MN) provides integrated real-time periprocedural imaging with enhanced threedimensional fluoroscopy (Figure 9). Distinct from augmented fluoroscopy technologies, fluoroscopic navigation uses advanced software algorithms and digital tomosynthesis reconstruction $(67,68)$ of multiple fluoroscopic images to enhance visualization and provide accurate three-dimensional modeling. A "local registration" feature employs fluoroscopy and a proprietary algorithm to update the virtual target location intraprocedurally so that the user can reposition the catheter as needed based on the actual nodule location, therefore adjusting for CT-to-body 

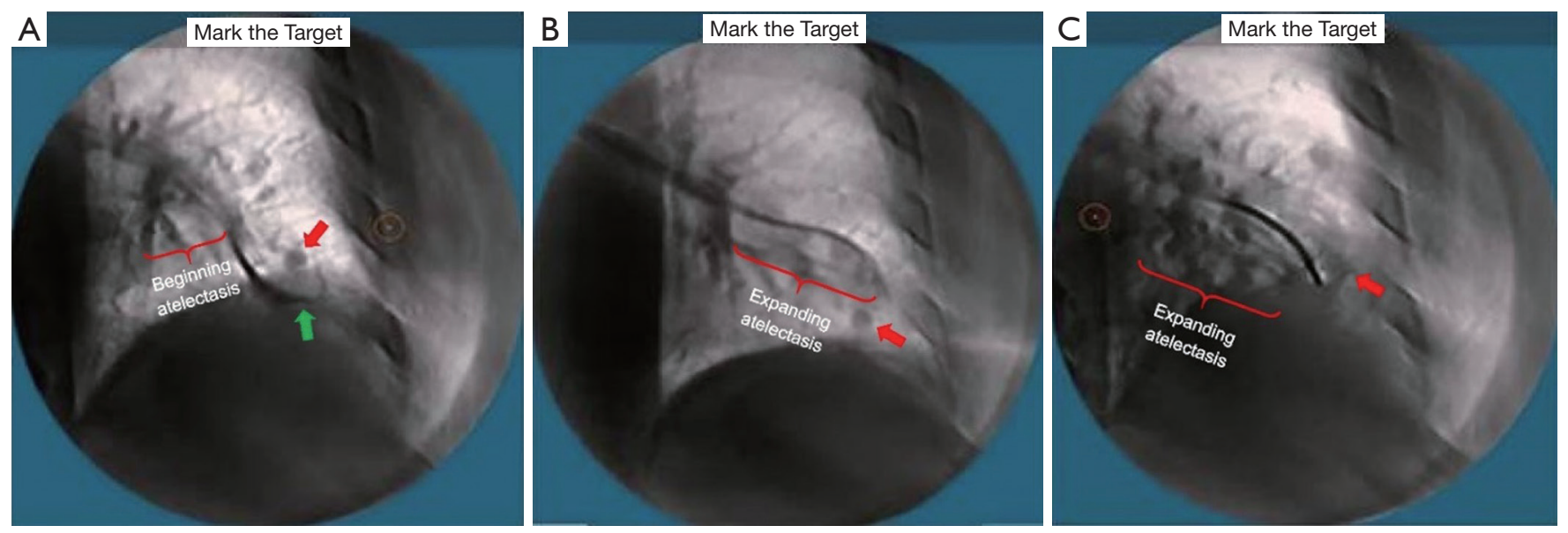

Figure 10 CT-to-body divergence and periprocedural evolution of airway configuration due to atelectasis during an ENB procedure using tomosynthesis-based fluoroscopic navigation. The virtual target is shown with a green arrow and the actual lung nodule is indicated with a red arrow. (A) Eight minutes after the start of electromagnetic navigation with the beginning of atelectasis. The virtual nodule is below the actual nodule, due to the lower position of the diaphragm in the preprocedural CT versus the ENB procedure. (B) Seventeen minutes after the start of navigation with continued atelectasis. The catheter has been moved to a different airway and the relative position of the nodule to the diaphragm has changed. (C) Twenty-eight minutes after the start of navigation. Operator was able to rotate the catheter and obtained a diagnosis. Images courtesy of Dr. Fabien Maldonado. All rights reserved. CT, computed tomography.

divergence (Figure 10). The first clinical study has recently been published, retrospectively comparing the new system (version 7.2) to the prior version (version 7.1). Diagnostic yield was significantly higher using fluoroscopic navigation (79.1\%) compared to standard navigation $(54.4 \%$; $\mathrm{P}=0.0019)$, despite a challenging cohort with $64 \%$ of lesions $<2 \mathrm{~cm}$, the absence of bronchus sign in $78 \%$, and the use of a conservative diagnostic yield definition (46). Of note, while rEBUS was used in all cases, diagnostic yield of fluoroscopic navigation was not impacted by the presence or absence of a concentric rEBUS view in multivariate models (46). A prospective, multicenter study (NCT03585959) has recently been completed to evaluate the three-dimensional location accuracy of this system as confirmed by CBCT (69) and is planned for presentation in October 2019.

The recently FDA-cleared ILLUMISITETM Platform (Medtronic) uses proprietary tomosynthesis-based fluoroscopic navigation and local registration combined with continuous intraprocedural guidance using a sensor embedded in the catheter tip. Studies are planned to evaluate the performance of this technology.

\section{Summary}

In summary, CT-to-body divergence is an important factor in the localization accuracy of all guided-bronchoscopy systems that rely on preprocedural planning CT scans. An awareness of the causes of CT-to-body divergence and the implementation of evolving best practice techniques can significantly minimize its impact and improve diagnostic yield. New advanced bronchoscopy systems with real-time three-dimensional imaging adjuncts appear to overcome divergence more effectively than other methods and promise to further improve diagnostic yield; however, additional clinical data are needed. Immediate introduction of optimized anesthesia protocols to overcome atelectasis may also significantly improve outcomes without requiring any additional capital investments.

\section{Acknowledgements}

The authors thank Dr. Fabien Maldonado for his helpful review.

Funding: None.

\section{Footnote}

Provenance and Peer Review: This article was commissioned by the Guest Editor (Kassem Harris) for the series "Interventional Pulmonology" published in fournal of 
Thoracic Disease. This article was sent for external peer review organized by the Guest Editor and the editorial office.

Conflicts of Interest: All authors have completed the ICMJE uniform disclosure form (available at http:// dx.doi. org/10.21037/jtd.2020.01.35). The series "Interventional Pulmonology" was commissioned by the editorial office without any funding or sponsorship. KB reports nonfinancial support from Medtronic; personal fees from Medtronic, Boston Scientific, BodyVision, Auris Health, Inc., Intuitive Surgical, Veracyte, Biodesix, Merit Medical Endotek, Johnson and Johnson outside the submitted work. MC reports non-financial support from Medtronic, personal fees from Medtronic during the conduct of the study. EF reports non-financial support from Medtronic during the conduct of the study; personal fees from Medtronic, Intuitive Surgical, Boston Scientific outside the submitted work. MAP reports non-financial support from Medtronic during the conduct of the study; personal fees from Medtronic, BodyVision, Intuitive Surgical, Philips, Biodesix, AstraZeneca, Johnson \& Johnson, United Therapeutics, Actelion, other from Inivata, Pfizer, Ambu, Boston Scientific outside the submitted work; and Wife is an employee of Medtronic.

Ethical Statement: The authors are accountable for all aspects of the work in ensuring that questions related to the accuracy or integrity of any part of the work are appropriately investigated and resolved.

Open Access Statement: This is an Open Access article distributed in accordance with the Creative Commons Attribution-NonCommercial-NoDerivs 4.0 International License (CC BY-NC-ND 4.0), which permits the noncommercial replication and distribution of the article with the strict proviso that no changes or edits are made and the original work is properly cited (including links to both the formal publication through the relevant DOI and the license). See: https://creativecommons.org/licenses/by-nc-nd/4.0/.

\section{References}

1. Khemasuwan D, Küpeli E, Mehta AC. Electromagnetic Navigation: A Review. Interventions in Pulmonary Medicine: Springer; 2018:281-97.

2. Folch EE, Pritchett MA, Nead MA, et al. Electromagnetic Navigation Bronchoscopy for Peripheral Pulmonary
Lesions: One-Year Results of the Prospective, Multicenter NAVIGATE Study. J Thorac Oncol 2019;14:445-58.

3. Raval AA, Amir L. Community hospital experience using electromagnetic navigation bronchoscopy system integrating tidal volume computed tomography mapping. Lung Cancer Manag 2016;5:9-19.

4. Rojas-Solano JR, Ugalde-Gamboa L, Machuzak M. Robotic Bronchoscopy for Diagnosis of Suspected Lung Cancer: A Feasibility Study. J Bronchology Interv Pulmonol 2018;25:168-75.

5. Fielding DIK, Bashirzadeh F, Son JH, et al. First Human Use of a New Robotic-Assisted Fiber Optic Sensing Navigation System for Small Peripheral Pulmonary Nodules. Respiration 2019;98:142-50.

6. Herth FJ, Eberhardt R, Sterman D, et al. Bronchoscopic transparenchymal nodule access (BTPNA): first in human trial of a novel procedure for sampling solitary pulmonary nodules. Thorax 2015;70:326-32.

7. Hogarth DK. Use of augmented fluoroscopic imaging during diagnostic bronchoscopy. Future Oncol 2018;14:2247-52.

8. Mehta AC, Hood KL, Schwarz Y, et al. The Evolutional History of Electromagnetic Navigation Bronchoscopy. Chest 2018;154:935-47.

9. Ost DE, Ernst A, Lei X, et al. Diagnostic yield and complications of bronchoscopy for peripheral lung lesions. Results of the AQuIRE Registry. Am J Respir Crit Care Med 2016;193:68-77.

10. Chen AC, Pastis NJ, Mahajan AK, et al. Multicenter, Prospective Pilot and Feasibility Study of Robotic Assisted Bronchoscopy for Peripheral Pulmonary Lesions [Abstract]. Am J Respir Crit Care Med. 2019;199:A7304.

11. Chaddha U, Kovacs SP, Manley C, et al. Robot-assisted bronchoscopy for lung nodule diagnosis: a pilot feasibility study. Am J Respir Crit Care Med 2019;199:A1266.

12. Eberhardt R, Kahn N, Gompelmann D, et al. LungPoint-a new approach to peripheral lesions. J Thorac Oncol 2010;5:1559-63.

13. Tamiya M, Okamoto N, Sasada S, et al. Diagnostic yield of combined bronchoscopy and endobronchial ultrasonography, under LungPoint guidance for small peripheral pulmonary lesions. Respirology 2013;18:834-9.

14. Hogarth D, Bhadra K, Whitten P, et al. A novel endobronchial fluoroscopic navigation and localization system: A summary of a multicenter LungVision trial. Chest 2018;154:880A-2A.

15. Whitten P. Augmented endobronchial fluoroscopic navigation and localization system: Integration of 
multimodalities and biopsy tools to increase diagnostic yield. Chest 2018;154:658A.

16. Pritchett M. Comparison of pulmonary nodule location between preprocedural ct and intra-procedural conebeam CT during guided bronchoscopy. J Thorac Oncol 2018;13:S403.

17. Bhadra K, Hogarth D. Novel augmented fluoroscopic imaging platform accuracy assessment during navigation bronchoscopy in simultaneous comparison with cone beam CT and radial EBUS. Chest 2018;154:870A-1A.

18. DiBardino DM, Yarmus LB, Semaan RW. Transthoracic needle biopsy of the lung. J Thorac Dis 2015;7:S304-16.

19. Rivera MP, Detterbeck F, Mehta AC. Diagnosis of lung cancer: the guidelines. Chest 2003;123:129S-36S.

20. Bhatt KM, Tandon YK, Graham R, et al. Electromagnetic Navigational Bronchoscopy versus CT-guided Percutaneous Sampling of Peripheral Indeterminate Pulmonary Nodules: A Cohort Study. Radiology 2018;286:1052-61.

21. Furukawa BS, Pastis NJ, Tanner NT, et al. Comparing Pulmonary Nodule Location During Electromagnetic Bronchoscopy With Predicted Location on the Basis of Two Virtual Airway Maps at Different Phases of Respiration. Chest 2018;153:181-6.

22. Casal RF, Sarkiss M, Jones AK, et al. Cone beam computed tomography-guided thin/ultrathin bronchoscopy for diagnosis of peripheral lung nodules: a prospective pilot study. J Thorac Dis 2018;10:6950-9.

23. Kolar P, Neuwirth J, Sanda J, et al. Analysis of diaphragm movement during tidal breathing and during its activation while breath holding using MRI synchronized with spirometry. Physiol Res 2009;58:383-92.

24. Chen A, Pastis N, Furukawa B, et al. The effect of respiratory motion on pulmonary nodule location during electromagnetic navigation bronchoscopy. Chest 2015;147:1275-81.

25. Eberhardt R, Anantham D, Ernst A, et al. Multimodality bronchoscopic diagnosis of peripheral lung lesions: a randomized controlled trial. Am J Respir Crit Care Med 2007;176:36-41.

26. Pickering EM, Kalchiem-Dekel O, Sachdeva A. Electromagnetic navigation bronchoscopy: a comprehensive review. AME Med J 2018;3:117.</jrn>

27. Yarmus LB, Arias S, Feller-Kopman D, et al. Electromagnetic navigation transthoracic needle aspiration for the diagnosis of pulmonary nodules: a safety and feasibility pilot study. J Thorac Dis 2016;8:186-94.

28. Flenaugh E, Mohammed K. Initial Experience Using 4D Electromagnetic Navigation Bronchoscopy System With
Tip Tracked Instruments For Localization of Peripheral Lung Nodules. Internet J Pulm Med 2016;18:1.

29. Semaan RW, Lee HJ, Feller-Kopman D, et al. Same-Day Computed Tomographic Chest Imaging for Pulmonary Nodule Targeting with Electromagnetic Navigation Bronchoscopy May Decrease Unnecessary Procedures. Ann Am Thorac Soc 2016;13:2223-8.

30. Thiboutot J, Lee HJ, Silvestri GA, et al. Study Design and Rationale: A Multicenter, Prospective Trial of Electromagnetic Bronchoscopic and Electromagnetic Transthoracic Navigational Approaches for the Biopsy of Peripheral Pulmonary Nodules (ALL IN ONE Trial). Contemp Clin Trials 2018;71:88-95.

31. Zhao YR, Heuvelmans MA, Dorrius MD, et al. Features of resolving and nonresolving indeterminate pulmonary nodules at follow-up CT: the NELSON study. Radiology 2014;270:872-9.

32. Lundquist H, Hedenstierna G, Strandberg A, et al. CTassessment of dependent lung densities in man during general anaesthesia. Acta Radiol 1995;36:626-32.

33. Strandberg A, Tokics L, Brismar B, et al. Atelectasis during anaesthesia and in the postoperative period. Acta Anaesthesiol Scand 1986;30:154-8.

34. Brismar B, Hedenstierna G, Lundquist H, et al. Pulmonary densities during anesthesia with muscular relaxation--a proposal of atelectasis. Anesthesiology 1985;62:422-8.

35. Rusca M, Proietti S, Schnyder P, et al. Prevention of Atelectasis Formation During Induction of General Anesthesia. Anesth Analg 2003;97:1835-9.

36. Coussa M, Proietti S, Schnyder P, et al. Prevention of Atelectasis Formation During the Induction of General Anesthesia in Morbidly Obese Patients. Anesth Analg 2004;98:1491-5, table of contents.

37. Pritchett MA, Schampaert S, de Groot JAH, et al. ConeBeam CT With Augmented Fluoroscopy Combined With Electromagnetic Navigation Bronchoscopy for Biopsy of Pulmonary Nodules. J Bronchology Interv Pulmonol 2018;25:274-82.

38. Brimacombe J. The advantages of the LMA over the tracheal tube or facemask: a meta-analysis. Can J Anaesth 1995;42:1017-23.

39. Sarkiss M. Anesthesia for bronchoscopy and interventional pulmonology: from moderate sedation to jet ventilation. Curr Opin Pulm Med 2011;17:274-8.

40. Hohenforst-Schmidt W, Zarogoulidis P, Huang H, et al. A New and Safe Mode of Ventilation for Interventional Pulmonary Medicine: The Ease of Nasal Superimposed High Frequency Jet Ventilation. J Cancer 2018;9:816-33. 
41. Webb TN, Bonta DV, Masters R, et al. Jet Ventilation Decreases Target Motion and Increases Yield of ENB Especially in Lesions With Negative Bronchus Sign. J Bronchology Interv Pulmonol 2020;27:14-21.

42. Knill RL. Control of breathing: effects of analgesic, anaesthetic and neuromuscular blocking drugs. Can J Anaesth 1988;35:S4-8.

43. Froese AB, Bryan AC. Effects of anesthesia and paralysis on diaphragmatic mechanics in man. Anesthesiology 1974;41:242-55.

44. Kleinman BS, Frey K, VanDrunen M, et al. Motion of the diaphragm in patients with chronic obstructive pulmonary disease while spontaneously breathing versus during positive pressure breathing after anesthesia and neuromuscular blockade. Anesthesiology 2002;97:298-305.

45. Kirov K, Motamed C, Dhonneur G. Differential Sensitivity of Abdominal Muscles and the Diaphragm to Mivacurium: An Electromyographic Study. Anesthesiology 2001;95:1323-8.

46. Aboudara M, Roller L, Rickman O, et al. Improved diagnostic yield for lung nodules with digital tomosynthesiscorrected navigational bronchoscopy: Initial experience with a novel adjunct. Respirology 2020;25:206-13.

47. Belanger AR, Burks AC, Chambers DM, et al. Peripheral lung nodule diagnosis and fiducial marker placement using a novel tip-tracked electromagnetic navigation bronchoscopy system. J Bronchology Interv Pulmonol 2019;26:41-8.

48. Faisal AH, Sopian AW, Tidi H. Electro-navigational bronchoscopy for bilateral peripheral lung lesions: A case report. Med J Malaysia 2017;72:241-3.

49. Sterman DH, Keast T, Rai L, et al. High yield of bronchoscopic transparenchymal nodule access realtime image-guided sampling in a novel model of small pulmonary nodules in canines. Chest 2015;147:700-7.

50. Oki M, Saka H, Asano F, et al. Use of an Ultrathin vs Thin Bronchoscope for Peripheral Pulmonary Lesions: A Randomized Trial. Chest 2019;156:954-64.

51. Ali EAA, Takizawa H, Kawakita N, et al. Transbronchial Biopsy Using an Ultrathin Bronchoscope Guided by Cone-Beam Computed Tomography and Virtual Bronchoscopic Navigation in the Diagnosis of Pulmonary Nodules. Respiration 2019;98:321-8.

52. Harzheim D, Sterman D, Shah PL, et al. Bronchoscopic Transparenchymal Nodule Access: Feasibility and Safety in an Endoscopic Unit. Respiration 2016;91:302-6.

53. Hohenforst-Schmidt W, Zarogoulidis P, Vogl T, et al. Cone Beam Computertomography (CBCT) in Interventional Chest Medicine - High Feasibility for Endobronchial
Realtime Navigation. J Cancer 2014;5:231-41.

54. Sobieszczyk MJ, Yuan Z, Li W, et al. Biopsy of peripheral lung nodules utilizing cone beam computer tomography with and without trans bronchial access tool: a retrospective analysis. J Thorac Dis 2018;10:5953-9.

55. Bowling MR, Brown C, Anciano CJ. Feasibility and safety of the transbronchial access tool for peripheral pulmonary nodule and mass. Ann Thorac Surg 2017;104:443-9.

56. Chen AC, Gillespie CT. Robotic Endoscopic Airway Challenge: REACH Assessment. Ann Thorac Surg 2018;106:293-7.

57. Mallow C, Lee H, Oberg C, et al. Safety and diagnostic performance of pulmonologists performing electromagnetic guided percutaneous lung biopsy (SPiNperc). Respirology 2019;24:453-8.

58. Lee HJ, Lerner AD, Coleman B, et al. Learning Electromagnetic Navigational Bronchoscopy and Percutaneous Transthoracic Needle Biopsy (LEAP): A Pilot Study. J Bronchology Interv Pulmonol 2019;26:55-61.

59. Grand DJ, Atalay MA, Cronan JJ, et al. CT-guided percutaneous lung biopsy: comparison of conventional CT fluoroscopy to CT fluoroscopy with electromagnetic navigation system in 60 consecutive patients. Eur J Radiol 2011;79:e133-6.

60. Oki M, Saka H, Ando M, et al. Ultrathin Bronchoscopy with Multimodal Devices for Peripheral Pulmonary Lesions. A Randomized Trial. Am J Respir Crit Care Med 2015;192:468-76.

61. Clinical Utility for Ion Endoluminal System (NCT03893539). ClinicalTrials.gov website. Available online: https://clinicaltrials.gov/ct2/show/NCT03893539. Updated May 23, 2019. Accessed June 17, 2019.

62. Tanner NT, Yarmus L, Chen A, et al. Standard Bronchoscopy With Fluoroscopy vs Thin Bronchoscopy and Radial Endobronchial Ultrasound for Biopsy of Pulmonary Lesions: A Multicenter, Prospective, Randomized Trial. Chest 2018;154:1035-43.

63. Ozgul G, Cetinkaya E, Ozgul MA, et al. Efficacy and safety of electromagnetic navigation bronchoscopy with or without radial endobronchial ultrasound for peripheral lung lesions. Endosc Ultrasound 2016;5:189-95.

64. Chen A, Chenna P, Loiselle A, et al. Radial probe endobronchial ultrasound for peripheral pulmonary lesions. A 5-year institutional experience. Ann Am Thorac Soc 2014;11:578-82.

65. Lau K, Spiers A, Pritchett M, et al. P1.05-06 Bronchoscopic Image-Guided Microwave Ablation of Peripheral Lung Tumours - Early Results. J Thorac Oncol 
2018;13:S542.

66. Evaluation of the Archimedes ${ }^{\mathrm{TM}}$ System for Transparenchymal Nodule Access 2 (EAST2) (NCT02867371). ClinicalTrials.gov website. Available online: https://clinicaltrials.gov/ct2/show/NCT02867371. Updated June 24, 2018. Accessed August 30, 2019.

67. Ferrari A, Bertolaccini L, Solli P, et al. Digital chest tomosynthesis: the 2017 updated review of an emerging application. Ann Transl Med 2018;6:91.

Cite this article as: Pritchett MA, Bhadra K, Calcutt M, Folch E. Virtual or reality: divergence between preprocedural computed tomography scans and lung anatomy during guided bronchoscopy. J Thorac Dis 2020;12(4):1595-1611. doi: 10.21037/ jtd.2020.01.35
68. Nelson G, Wu M, Hinkel C, et al. Improved targeting accuracy of lung tumor biopsies with scanning-beam digital X-ray tomosynthesis image guidance. Med Phys 2016;43:6282.

69. superDimension ${ }^{\mathrm{TM}}$ Navigation System Version 7.2 With Fluoroscopic Navigation Technology (NCT03585959). ClinicalTrials.gov website. Available online: https:// clinicaltrials.gov/ct2/show/NCT03585959. Updated February 18, 2019. Accessed May 1, 2019. 